\title{
Anne Berman (1889-1979), une «simple secrétaire» du mouvement psychanalytique français?
}

Rémy Amouroux

\section{Summary}

This article is focused on the figure of personal secretary in the history of science with the example of Anne Berman (1889-1979) who was, between 1933 and 1962, the secretary for the psychoanalyst Marie Bonaparte (18821962). Berman was not a psychoanalyst and psychoanalytic historiography considers her as a minor figure. However, her career as a personal secretary and her role in the French psychoanalytic movement should be considered in conjunction with her involvement with the feminist movement. This pharmacist by training has indeed played a prominent role within the Soroptimist, which was a movement that championed the professional interest of women and prides female excellence. In the case of Berman, the status of personal secretary did not enable her to gain lasting recognition by psychoanalysts, but only a weak and fragile legitimacy.

Keywords: Anne Berman, Personal Secretary, Translation, Psychoanalysis

Rémy Amouroux, Université de Lausanne, Geopolis, Institut de Psychologie, CH-1015 Lausanne (remy.amouroux@unil.ch) 


\section{Résumé}

Cet article s'intéresse à la figure du secrétaire particulier dans l'histoire des sciences à travers l'exemple d'Anne Berman (1889-1979) qui fut la secrétaire de la psychanalyste Marie Bonaparte (1882-1962) de 1933 à 1962. Berman n'est pas psychanalyste et l'historiographie psychanalytique en fait donc un personnage de second plan. Cependant, sa carrière de secrétaire particulière et son rôle au sein du mouvement psychanalytique français gagnent à être mis en parallèle avec son engagement au sein d'un réseau de femmes. Cette pharmacienne de formation a en effet joué un rôle considérable au sein des Soroptimist, un mouvement qui défend les intérêts professionnels des femmes et prône l'excellence féminine. Dans le cas d'Anne Berman, le statut de secrétaire particulier n'a pas permis d'accéder à une reconnaissance durable par les psychanalystes, mais seulement à une forme de légitimité partielle et fragile.

\section{Introduction}

L'histoire de la psychanalyse est essentiellement centrée sur la figure de Sigmund Freud. Cette focalisation a été critiquée et il existe des propositions historiographiques alternatives pertinentes. ${ }^{1}$ L'une des manières de procéder pour pallier cet écueil consiste à opter pour une «histoire par le bas». Cela implique de prendre au sérieux des individus habituellement considérés comme «modestes» ou comme «profanes» et pas seulement les «grands hommes» qui sont célébrés par la discipline. A ce sujet, les travaux initiés par Roy Porter sur le point de vue des patients ont permis de mieux comprendre l'histoire des pratiques et des savoirs "psy».2

Qu'en est-il des secrétaires particuliers? S'agit-il d'une de ces catégories d'individus considérés comme mineure dont nous parlions précédemment? En effet, les secrétaires particuliers, par le poste qu'ils occupent, sont à la fois très proches et très éloignés des acteurs officiels du mouvement. Proches parce qu'ils accompagnent leur «employeur» au quotidien et qu'ils ont tout loisir d'observer ce que l'on réserve habituellement aux plus intimes. Eloignés car le statut de secrétaire implique une hiérarchie naturelle dans la relation qui, dès lors, ne saurait être comparable à celle que l'on entretient avec ses

1 Citons notamment le célèbre livre d'Henri Ellenberger (1994) ou encore les travaux plus récents de Lydia Marinelli et d'Andreas Mayer (2009) qui tous les deux brisent le mythe d'un Freud isolé, se frayant un passage dans un milieu hostile.

2 Porter 1985. 
pairs. C'est donc par définition un acteur invisible - ou en tout cas peu audible - dont le rôle est difficile à évaluer.

Dans ce travail, nous étudierons en détail la trajectoire professionnelle d'Anne Berman qui fut la secrétaire de la psychanalyste Marie Bonaparte (1882-1962) de 1933 à 1962. Berman n'est pas psychanalyste mais elle est une femme éduquée, docteure en pharmacie et polyglotte. Elle est aussi engagée dans la vie publique notamment au sein du mouvement Soroptimist qui défend les intérêts professionnels des femmes et prône l'excellence féminine. Dans l'historiographie psychanalytique, elle est surtout connue pour ses traductions en français de l'œuvre de Sigmund Freud qui, après avoir fait référence, furent progressivement abandonnées. Berman serait une pionnière dont les choix de traductrice révèlent le statut d'amateur. Pourtant, en suivant au plus près la carrière de Berman, on peut aboutir à une perception différente et à une tout autre interprétation quant au statut de ses traductions.

A travers cet exemple, il s'agira d'explorer la figure du secrétaire particulier dans l'histoire des sciences, et plus particulièrement dans l'histoire des sciences du psychisme. Je tenterai de montrer que, dans le cas de Berman, il ne s'agit pas d'un simple travail d'exécutante sans aucune autonomie, mais bien d'une manière originale de participer au débat scientifique. Pour ce faire, je commencerai par m'interroger sur la fonction de secrétaire dans l'histoire des sciences, puis je m'intéresserai aux différentes carrières de Berman tant dans le milieu de la psychanalyse française qu'au sein d'un réseau social féminin, enfin je développerai plus particulièrement son travail contesté de traductrice de textes psychanalytiques.

\section{La figure du secrétaire particulier}

Affranchi, éminence grise ou simple employé de bureau?

C'est à Tacite, l'historien et sénateur romain du premier siècle, que l'on attribue l'une des premières définitions d'une fonction proche de ce que l'on appelle aujourd'hui le secrétaire particulier. Il rapporte en effet que les patriciens romains avaient recours aux services d'«affranchis employés aux affaires confidentielles». ${ }^{3} \mathrm{~L}$ '《affranchi» désigne ici un esclave auquel on a octroyé la liberté, ce qui préfigure le rapport hiérarchique entre le secrétaire et celui qui l'emploie. Ce serviteur s'occupe par ailleurs des «affaires confidentielles» de son employeur ce qui implique qu'il s'agit d'un travailleur de l'ombre qui exerce en «service commandé».

3 Blanc-Sanchez 1992. 
Dans l'historiographie, la majorité des travaux disponibles sur les secrétaires particuliers portent sur des acteurs politiques de l'Ancien Régime dont le rôle a été sous-estimé. Ainsi, pour citer un exemple parmi d'autres, Poisson $^{4}$ a mis en évidence le rôle crucial des secrétaires-interprètes de l'ambassadeur de France à Soleure en Suisse dans la seconde partie du XVII ${ }^{\mathrm{e}}$ siècle. Ici, c'est le rôle de conseiller personnel qui semble déterminant. L'exemple le plus célèbre est probablement celui du Père Joseph, le secrétaire du cardinal de Richelieu. François Leclerc du Tremblay, plus connu sous le nom de Père Joseph, fut un moine capucin proche confident du cardinal. ${ }^{5}$ Il doit son surnom «d'Eminence grise» à la couleur de sa robe de bure et au pouvoir dont il aurait joui en tant que proche conseiller de «son Eminence», le cardinal de Richelieu.

Enfin, au milieu du XIX ${ }^{\mathrm{e}}$ siècle, la diffusion de la machine à écrire et du téléphone ainsi que des modifications de la structure du marché de l'emploi ont participé à l'émergence d'une nouvelle profession: le secrétaire de bureau. ${ }^{6}$ Cette activité, initialement réservée aux hommes, a rapidement évolué et s'est féminisée. Le terme de secrétaire désigne alors des femmes employées de bureau chargées de rédiger le courrier mais aussi d'aider à l'organisation générale et à la bonne tenue des affaires de leur employeur. Comme le note Pinto, ${ }^{7}$ outre ses compétences techniques, la secrétaire est une employée qui doit s'appuyer sur ses qualités féminines relationnelles pour «enchanter»-adoucir - les relations dans un milieu où la concurrence «virile» est la règle.

\section{Le secrétaire de savant}

Il y a peu de travaux dans l'historiographie portant spécifiquement sur les secrétaires de savants. Il s'agit généralement d'hommages à des personnes auxquelles les historiens sont redevables car ils ont préservé tout ou partie de l'œuvre de leur maître. Ainsi, l'historien de la physique Gerald Holton souligne sa gratitude pour Helen Dukas, la secrétaire d'Albert Einstein pendant près de trente ans, à qui on doit la préservation d'une partie des archives du célèbre physicien. ${ }^{8}$ Dans la même veine, Léonie Razet, qui fut la secrétaire de Marie Curie, est souvent créditée dans l'historiographie pour le rôle «important» qu'elle a joué. ${ }^{9}$ Dans ces deux cas, faute d'informations

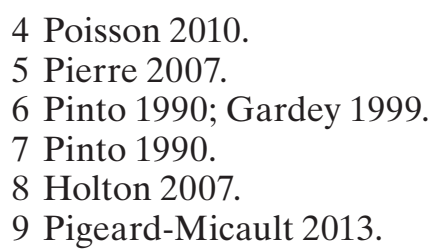


précises, il semblerait que leur contribution se limite à la gestion habile des affaires courantes durant leur vie professionnelle et à la constitution des archives après le décès de leur ancien employeur. Ici encore, la féminisation de cette profession est importante à noter. En effet, ces proches collaborateurs sont le plus souvent de proches collaboratrices qui évoluent dans un milieu où ce sont les hommes qui dominent sans partage le monde académique. Pour se faire une place dans ce domaine, les femmes ont fréquemment dû se limiter à certains domaines considérés comme féminins, ou encore accepter d'avoir une reconnaissance limitée des milieux académiques. ${ }^{10}$ Ainsi, à défaut de pouvoir exercer comme des actrices légitimes, certaines de ces femmes ont investi des lieux à l'écart des institutions savantes, faisant par exemple de leur foyer un laboratoire pour l'observation des enfants..$^{11}$ De même, ces secrétaires de savants ont pratiqué une forme de science en dehors des milieux académique, à la frontière de l'amateurisme, en assistant leur employeur dans les différentes tâches requises par la recherche scientifique. Cet amateurisme peut d'ailleurs peut-être même être vu comme un choix raisonnable ${ }^{12}$ compte tenu du contexte fort peu favorable au développement des carrières académiques féminines.

\section{Le secrétaire du «psy»}

Otto Rank, s'il ne fut pas réellement le secrétaire particulier de Freud, est devenu à l'âge de 22 ans, en 1906, le secrétaire de la Société Psychanalytique de Vienne. ${ }^{13}$ Freud a donné à Rank une place toute particulière dans le mouvement psychanalytique. Il lui paie ses études universitaires et le nomme secrétaire de la première société psychanalytique. De 1910 à 1915, Rank est chargé d'établir le compte rendu détaillé - les «Minutes» - des soirées du mercredi chez Freud. Ce n'est pas un simple titre honorifique mais un travail qui va lui permettre d'entreprendre des études universitaires. Cependant, Rank obtient en 1912 un doctorat en psychologie et a exercé en tant que psychanalyste avant de se brouiller avec le fondateur de la psychanalyse. A ce titre, s'il fut un temps un protégé et un proche collaborateur de Freud, il est par la suite devenu un membre à part entière de la société savante. Ainsi, il ne correspond pas à proprement parler à la définition de secrétaire particulier que nous évoquions précédemment. Le cas de Gloria Gonzalez, la secrétaire particulière de Jacques Lacan de 1948 à sa mort, est probablement

10 Carroy/Edelman/Ohayon/Richard 2005.

11 Oertzen 2013.

12 Pomata 2013.

13 Lieberman 1985. 
plus intéressant. ${ }^{14}$ D'abord au service de Sylvia, la fille de Lacan, Gloria va progressivement devenir une proche collaboratrice du théoricien du «retour à Freud». De plus, Gloria semble avoir joué un rôle actif auprès des analysants de Lacan: «Quand il reçoit ses patients, elle ouvre la porte puis surveille du coin de l'œil les déplacements de tous et de chacun [...] Elle calme les agités, console les anxieux ou accompagne dans l'escalier les candidats à la dépression. ${ }^{15}$ Il y a en effet beaucoup de témoignages de son rôle «apaisant». ${ }^{16}$ En plus de Gloria qui a joué, auprès de Lacan, davantage le rôle d'assistante personnelle que celui de secrétaire, on peut aussi mentionner Maria Pierrakos. Cette dernière fut pendant douze ans sa sténotypiste. Elle fut notamment chargée de retranscrire les fameux séminaires du maître tout en poursuivant des études de psychologie et une formation d'analyste. ${ }^{17} \mathrm{Ces}$ exemples semblent donc bien corroborer l'hypothèse que certaines de ces secrétaires de savants (ici de psychanalystes) ont pu bénéficier - directement ou indirectement - de leur position pour faire carrière et passer du statut de collaborateur à celui de pair. Comme nous allons le voir, le cas de Berman va dans le même sens.

\section{Anne Berman, la Société Psychanalytique de Paris et le mouvement Soroptimist}

\section{Une secrétaire dévouée}

Comment cette pharmacienne est-elle devenue la secrétaire d'une des pionnières de la psychanalyse en France? On dispose de peu de travaux portant spécifiquement sur Anne Berman. ${ }^{18}$ Le 10 janvier 1927 où Berman devient la toute première adhérente de la Société Psychanalytique de Paris (SPP). Pourquoi cette femme qui n'est ni psychanalyste, ni médecin, ni psychologue s'est-elle affiliée à ce groupe? Berman est proche de plusieurs pionniers de la psychanalyse française, notamment d'Odette et de Henri Codet, mais aussi et surtout d'Adrien Borel avec qui elle entretint une liaison amoureuse pendant plusieurs années. Au début de l'année 1930, Anne Berman entreprend une psychanalyse avec Marie Bonaparte qui se terminera à la fin de l'année 1932. Au cours de l'été 1933, Berman devient officiellement la secrétaire de la princesse de Grèce, succédant ainsi à un jeune homme du nom de Sello

14 Roudinesco 1994.

15 Roudinesco 1994, 305.

16 Godin 1990; Haddad 2002; Rey 1989.

17 Pierrakos 2003.

18 Bourgeron 2005. 
Frenkel. Cette activité, loin d'être un hobby intellectuel, va progressivement occuper une bonne partie de l'emploi du temps de Berman. En effet, s'il s'agit d'abord uniquement de gérer l'abondante correspondance de Bonaparte, Berman va petit à petit étendre son champ de compétences: ses talents de polyglotte en font une assistante de choix pour les traductions de Freud sur lesquelles travaille Bonaparte, mais c'est surtout sa connaissance fine du milieu psychanalytique qui va faire de Berman une sorte de conseillère personnelle. Bonaparte étant souvent en voyage, Berman la représente à Paris et l'informe presque quotidiennement de la vie tumultueuse du mouvement psychanalytique français. Cet engagement implique nécessairement une forme substantielle de rémunération. Elles ne parlent jamais directement de cela, mais tout semble indiquer que Bonaparte, qui a la réputation d'être généreuse, salarie Berman.

De 1933 à 1962, elles entretiennent toutes les deux une abondante correspondance où on peut suivre l'histoire du mouvement psychanalytique français - notamment celle de la Société Psychanalytique de Paris - sous un angle original. Ce groupe de pionniers, créé en 1926, est à l'origine du développement de la psychanalyse en France. Composé d'hommes, médecins de surcroit, c'est un milieu où la place d'une femme sans diplôme universitaire, comme Bonaparte, ne va pas de soi. Sa fortune, son réseau social, son amitié avec la famille Freud, et son indépendance d'esprit vont lui permettre de jouer un rôle décisif. Cette descendante de l'Empereur mariée au prince de Grèce et du Danemark, va réussir à se faire une place de premier ordre et à participer à la mise en minorité de ceux qui souhaitent que le mouvement français prenne ses distances avec la stricte orthodoxie freudienne. ${ }^{19}$ D'une manière générale, loin d'être une simple mécène, Marie Bonaparte a durablement marqué la première génération de psychanalystes français. Elle est alors reconnue pour ses travaux sur la sexualité féminine, sa conception d'une psychanalyse ancrée dans les sciences naturelles ou encore son engagement pour l'exercice de la psychanalyse par les non-médecins. Berman est alors aux premières loges de ce débat intellectuel. Elle n'y participe pas directement mais y apprend sûrement beaucoup et échange fréquemment sur ces sujets. Si elle n'a jamais été une théoricienne ou une praticienne du mouvement, Berman est cependant à l'origine d'un nombre très important de traductions de textes de psychanalystes. Il est probable que Bonaparte, constatant ses talents linguistiques dans ses fonctions de secrétariat, l'ait encouragé à traduire des textes. Rien ne semble indiquer en effet que Berman ne dispose d'une formation professionnelle en langues. En outre, elle s'oc-

19 Ohayon 1999. 
cupe dès 1934 - l'année de sa création - du secrétariat de l'Institut de Psychanalyse et devient secrétaire de la Revue Française de Psychanalyse (RFP) de 1948 à 1969. Il est étonnant que cette collaboratrice personnelle d'un des membres de la SPP ait pu occuper ces fonctions qui semblent plutôt devoir être réservées à un membre titulaire de la société. Berman semble avoir occupé un statut flou entre celui de collaboratrice et de membre à part entière. Elle est d'ailleurs nommée membre titulaire de la SPP en 1949. Cette nomination est surprenante car, contrairement à tous les autres membres titulaires, Berman n'est pas psychanalyste. Elle a certes été analysée mais n'a pas suivi une formation de psychanalyste. Pourtant, il ne semble pas qu'il s'agisse d'une erreur car Anna Freud lui écrit à cette occasion une lettre de félicitation. ${ }^{20}$ Malgré ce message enthousiaste, dès l'année suivante et sans que l'on puisse trouver d'élément d'explication, son nom disparaît de la liste des membres titulaires de la SPP. Elle continuera à travailler auprès de Bonaparte jusqu'au décès de cette dernière.

\section{Une militante de l'excellence féminine}

Cette première série d'informations biographiques fait cependant l'impasse sur tout un pan de la vie professionnelle de Berman: sa carrière au sein du mouvement des Soroptimist. Car avant d'être la secrétaire personnelle de Bonaparte, Berman est d'abord une femme éduquée et ambitieuse. Ainsi, après avoir obtenu une licence de lettres, elle fait des études de pharmacie, soutient une thèse en botanique sur la «famille des Borraginacées» ${ }^{21}$ et entre à l'hôpital Sainte-Anne en qualité de pharmacienne-adjointe dans le laboratoire d'Edouard Toulouse, où elle restera jusqu'en 1924. Elle acquiert alors la pharmacie Souffron rue de Miromesnil à Paris où elle va exercer pendant de nombreuses années. A la fin de son analyse avec Bonaparte, elle hésite entre un poste de pharmacien des prisons et un poste de pharmacien-chef à l'hôpital de Grenoble. Mais, à lire leur correspondance, on comprend que ces deux postes ne l'intéressent pas suffisamment, financièrement et intellectuellement. C'est à ce moment là - en 1933 - qu'elle devient la secrétaire particulière de Bonaparte. En 1940, elle est interdite d'exercer en tant que pharmacienne, probablement à cause de ses origines juives. Elle ne se séparera pourtant de sa pharmacie qu'au sortir de la Deuxième Guerre mondiale. Par ailleurs, elle s'investit dans différents domaines et sera déléguée des pharmaciens à la chambre syndicale. Cependant, c'est surtout son engage-

20 Lettre d'Anne Berman à Marie Bonaparte du 10.12.1949, Bibliothèque Nationale de France (BNF), fonds Marie Bonaparte.

21 Berman 1926. 
ment chez les Soroptimist qu'il semble important d'explorer ici. Soroptimist est la contraction de l'expression latine «sorores ad optimum», que l'on peut traduire par «sœurs pour le meilleur». Cette organisation est fondée en 1921 par Violet Richardson Ward aux Etats-Unis dans l'état de Californie. Elle prône l'excellence intellectuelle, morale et professionnelle des femmes. C'est par l'intermédiaire de Suzanne Noël, une femme médecin, que le premier «club» français - et européen par la même occasion - voit le jour à Paris en 1924. ${ }^{22}$ Pendant la Première Guerre mondiale, Noël s'initie aux techniques de la chirurgie réparatrice auprès des «gueules cassées» et, par la suite, s'engage auprès des suffragettes. ${ }^{23} \mathrm{Au}$ sortir de la guerre, elle devient une pionnière de la chirurgie esthétique qu'elle conçoit comme une pratique progressiste. ${ }^{24}$ Parmi les autres membres du mouvement Soroptimist en France, on trouve des femmes militantes politiques comme par exemple Cécile Brunschvicg qui, en 1936, est l'une des trois premières femmes à siéger dans un gouvernement français, ou encore Germaine Poinso-Chapuis qui fut en 1947 la première femme ministre en France. Il y a aussi des artistes comme la poétesse Anna de Noailles et la couturière Jeanne Lanvin, mais aussi des juristes comme Marcelle Kraemer-Bach et Yvonne Netter, ou encore des psychanalystes comme Blanche Reverchon-Jouve ou Juliette Favez-Boutonnier. Toutes ont en commun d'être des pionnières ou d'exceller dans leur exercice professionnel. Elles ne sont cependant pas toutes célèbres. Ainsi, dans le numéro du bulletin français des Soroptimist où se présente Anne Berman en 1928, on trouve aussi une Madame Jouannin qui possède des «dons exceptionnels d'industrielle» ${ }^{25}$ dans le secteur émergent du commerce des réfrigérateurs, ou encore mademoiselle Terrier qui est traductrice de l'anglais. Anne Berman va jouer un rôle non négligeable au sein des Soroptimist françaises. La consultation du bulletin français du mouvement montre en effet qu'elle est fréquemment présente aux réunions et qu'elle a participé activement à la vie du groupe. Elle a d'ailleurs occupé dans les années 1950 les postes de secrétaire et de vice-présidente de l'Union Nationale Française des Soroptimist. A ce titre, son engagement dans le mouvement psychanalytique français peut apparaître comme secondaire comparé à celui dont qu'elle a occupé au sein des Soroptimist. En effet, alors qu'elle

22 Jacquemin 1988.

23 Guirimand 2005.

24 Suzanne Noël (Noël, 1926) conçoit la chirurgie esthétique comme une «thérapeutique progressiste» qui s'adresse en particulier aux femmes qui ne souhaitent pas renoncer à leur apparence corporelle. Elle propose ainsi ces interventions autant aux femmes aisées et privilégiées qui pensent le physique est un atout dans leur exercice professionnelle, à des résistantes qui souhaitent changer de visage pendant l'occupation, ou encore aux rescapées des camps de concentration dont le corps a été ravagé par la guerre.

25 Soroptimist-Club, 1928. 
est une actrice légitime et reconnue de l'Union féminine des intérêts professionnels, son statut au sein de la SPP est plus flou. Certes, elle y occupe certaines fonctions assez importantes comme celle de secrétaire de la RFP ou de l'Institut de psychanalyse, mais elle reste l'employée de Marie Bonaparte et n'a jamais été considérée comme une «véritable» psychanalyste.

\section{Traductions savantes et traductions amateurs}

Une traductrice «pour le meilleur»

L'engagement de Berman au sein du mouvement psychanalytique français semble être une mise en pratique à la lettre de la philosophie générale des Soroptimist. Comme d'autres membres qui furent des pionnières dans leur propre domaine, Berman est, au sujet des traductions de Freud, une «sœur pour le meilleur» pour reprendre la devise de son club. C'est d'ailleurs comme «traductrice» de «travaux scientifiques et philosophiques» ${ }^{26}$ qu'elle se présente dans le registre des membres des Soroptimist françaises à partir de 1947. Comme l'a écrit Alain de Mijolla, Anne Berman «[...] va devenir la traductrice attitrée de Freud en français, car elle bénéficie de l'appui inconditionnel de Marie Bonaparte». ${ }^{27}$ Une analyse de la RFP entre 1927 et 1962 permet de confirmer l'affirmation de Mijolla. C'est Anne Berman qui arrive en tête du nombre des traductions, un peu moins d'un quart - 15 sur les 67 traductions de la période - des articles traduits. Outre les productions de Berman, on trouve principalement celles de Marie Bonaparte (12 traductions), celles d'Henri Hoesli (10 traductions). Hoesli est un protégé de Marie Bonaparte. Ce Suisse originaire du canton de Glaris fut en effet le précepteur de Pierre de Grèce, le fils de la princesse. Cette dernière l'a par la suite soutenu financièrement dans son projet d'entreprendre des études universitaires de médecine à Paris. ${ }^{28}$ Hoesli deviendra par la suite membre de la SPP. En contrôlant les traductions, et notamment en s'adjoignant les services de proches collaborateurs qui peuvent faire ce travail à sa place, Bonaparte peut défendre et imposer sa propre conception de la psychanalyse. Pour Berman et Hoesli, c'est aussi un moyen d'obtenir une reconnaissance liée au prestige de voir leur nom associé à celui de Freud. D'ailleurs, sans sous-estimer le rôle de Bonaparte, le travail de Berman est particulièrement conséquent en lui-même: sur la période qui nous intéresse, celle-ci peut être

26 Soroptimist-Club, 1947.

27 Mijolla 1991, 253.

28 Bertin 1982. 
considérée comme «la» traductrice officielle française car c'est elle qui signe, ou co-signe, le plus de traductions dans la RFP et ailleurs. Ainsi, son travail est suffisamment reconnu pour que ses collègues de la SPP lui remettent un prix qui récompense l'ensemble de ses traductions. Elle reçoit en effet en 1964 le prix Maurice-Bouvet dont elle est d'ailleurs la seule récipiendaire à ne pas être psychanalyste.

\section{Le marché convoité des traductions de Freud}

L'hégémonie de Bonaparte et Berman va progressivement être remise en question. Au sortir de la Deuxième Guerre mondiale, l'influence de la princesse n'est plus la même. En effet, Marie Bonaparte s'est exilée pendant plusieurs années en Afrique du Sud et, lorsqu'elle revient en France, le mouvement psychanalytique français est à l'aube d'une série de bouleversements comme la scission de $1953 .{ }^{29}$ Les membres fondateurs de la SPP se font rares, beaucoup sont décédés ou ont quitté la France. De nouveaux acteurs apparaissent et les traductions vont devenir l'un des enjeux notoires de cette reconfiguration des pouvoirs et des influences de chacun. Ainsi, Daniel Lagache, un médecin psychiatre normalien à l'origine de la première licence de psychologie en France, recommande à Anna Freud certaines personnes pour des traductions. ${ }^{30}$ De même, Maryse Choisy, une journaliste et écrivaine qui s'est intéressée de très près la psychanalyse notamment en créant la revue Psyché en 1947, contacte elle aussi la fille de Freud pour s'enquérir de la possibilité de traduire certains textes. ${ }^{31}$ L'attitude de Berman au sujet est tout à fait éloquente. Apprenant notamment que Lagache souhaite introduire de nouveaux candidats pour les traductions, elle écrit à la princesse:

Mais pourquoi diable confier des travaux semblables à des personnes qui ne connaissent ni la psychanalyse, ni le français? Ne pourrait-on réserver les droits de traductions à vousmême et à moi? 32

Assez rapidement ces candidats contactent directement des maisons d'édition avec des projets précis. En réponse à des demandes insistantes des Presses Universitaires de France, Berman se montre tout aussi catégorique:

«J'ai dit et je suppose que vous m'approuvez qu'Anna Freud et vous même ne consentiriez pas à ce qu'une autre que moi traduisent les ouvrages comme certaines semblent le proposer. $^{33}$

29 Ohayon 1999.

30 Lettre d'Anne Berman à Anna Freud du 14.01.1949, fonds Anna Freud, Library Of Congress (LOC).

31 Lettre de Maryse Choisy à Anna Freud du 22.12.1946, LOC.

32 Lettre d'Anne Berman à Marie Bonaparte du 19.07.1947, BNF.

33 Lettre d'Anne Berman à Marie Bonaparte du 25.01.1948, BNF. 
A la même période elle échange fréquemment avec Anna Freud pour la traduction française d'articles et d'ouvrages. Elle lui rend notamment visite à Londres à l'occasion de réunions organisées par les Soroptimist. Pourtant, malgré les affirmations véhémentes de Berman, il n'est plus possible de continuer à contrôler l'ensemble des traductions françaises. Par exemple, fin 1950, Paul Jury écrit à Anna Freud pour lui dire qu'avec Ernest Fraenkel il a traduit Inhibition, symptôme et angoisse de Freud ${ }^{34}$ et qu'il souhaite savoir ses conditions concernant les droits d'auteur. ${ }^{35}$ Berman et la princesse sont alors en train de traduire ce texte mais, mises devant le fait accompli, elles se résolvent à abandonner leur projet, du moins en apparence. Une fois la traduction imprimée, Bonaparte et Berman posent leur véto: le texte de Jury est trop mauvais pour être mis en vente. Ce dernier ne compte pas se laisser faire et menace les PUF de procès. Berman est chargée d'établir la liste des erreurs de traduction de Jury. ${ }^{36}$ En octobre 1953, après négociation, le livre est mis en vente avec une feuille d'erratum. ${ }^{37}$ Comme cet exemple l'illustre, le contexte a changé: la princesse, qui décédera moins de dix ans plus tard, n'a plus la même influence qu'auparavant. Après la disparition de la princesse, Berman n'est plus la secrétaire de personne. Elle devient alors un personnage au statut hybride qui, sans être psychanalyste, joue pendant encore quelques années un rôle au sein de la SPP.

\section{Une traductrice «historique»}

Berman ne peut plus imposer ses choix comme avant. Ainsi en 1965, alors qu'elle doit corriger une traduction de Freud effectuée par Rudolph Loewenstein, elle se heurte à une demande de Lagache qui l'enjoint expressément à reconsidérer ses choix de traductrice. Entre-temps, Lagache est devenu professeur et responsable de collection aux PUF:

Je suis en désaccord avec le professeur Lagache à propos du mot Trieb qu'il veut partout traduire par pulsion. Par exemple dans le cas de ce qu'on a jusqu'ici appelé «instinct de vie et instinct de mort », il désire (il exige même) qu'on dise «pulsion de vie et pulsion de mort». A moi, la «pulsion» ne me semble pas une chose permanente. ${ }^{38}$

Berman n'arrivera pas à défendre sa cause. Pire, à compter de cette période, ses traductions vont progressivement passer du statut de référence obligée à celui de contre-exemple. Entre temps, la psychanalyse française évolue très

34 Freud 1951.

35 lettre de Paul Jury à Anna Freud du 14.12.1950, fonds John Rodker, Harry Ransom Center3.

36 Lettre des PUF à Marie Bonaparte du 30.5.1952, BNF.

37 Lettre des PUF à Marie Bonaparte du 9.10.1953, BNF.

38 Lettre d'Anne Berman à Rudolph Loewenstein du 12.5.1965, LOC. 
rapidement. Ces changements vont notamment se traduire par une attitude différente vis-à-vis de l'œuvre de Freud. Je pense ici à Lacan et à son «retour à Freud», ${ }^{39}$ ou encore au Vocabulaire de la psychanalyse ${ }^{40}$ publié par Jean Laplanche et Jean-Bertrand Pontalis en 1967. Cette attention nouvelle au texte aboutira au projet des œuvres complètes de Freud en français. Au milieu des années 1980, Laplanche entreprend avec un groupe de psychanalystes et de germanistes de publier un équivalent français à la Standard Edition de James Stratchey. ${ }^{41}$ Laplanche est critique vis-à-vis de ceux qu'il appelle les «traducteurs des commencements»:

Personne, plus que Freud n'a été mis à mal par ses traducteurs. Je n'entends pas ici revenir aux moqueries - désormais historiques - dont on n'accablait naguère ces traducteurs non moins historiques, Anne Berman ou Serge Jankélévitch. ${ }^{42}$

Le groupe de Laplanche reproche tout particulièrement à Bonaparte et Berman les choix de termes contestables qui ont entraîné, selon eux, des contresens importants. Odile Bourguignon qualifie ainsi les traductions de Bonaparte «d'acclimatisantes» ${ }^{43}$ et «d'éthnocentriques». ${ }^{44,45}$ Mais, sans entrer dans un débat qui dépasse l'objet de ce travail, la traduction de Freud par Laplanche et ses étonnants néologismes - «désaide» ou encore «désirance» - rendent-ils réellement Freud plus accessible au lectorat français? La multiplication des nouvelles traductions depuis le passage en 2010 dans le domaine public de l'œuvre de Freud, montre en tout cas que le débat n'est pas clos. ${ }^{46}$

Ainsi, si la marginalisation des traductions de Berman s'explique en partie par les transformations du champ psychanalytique français, son statut d'amateur a largement accéléré le phénomène. Après la mort de Bonaparte, Berman ne dispose plus des appuis suffisants pour défendre son travail. Malgré sa brillante ascension au sein du milieu psychanalytique, elle reste une secrétaire particulière sans pratique de la psychanalyse et ne sera donc jamais considérée comme une actrice légitime du mouvement.

39 Roudinesco 1994.

40 Laplanche/Pontalis 1967.

41 Amouroux 2011.

42 Laplanche 1984.

43 Bourguignon 198911.

44 Bourguignon 1989, 22.

45 Ce faisant, elle se réfère aux travaux du théoricien français de la traduction Antoine Berman (1942-1991) qui, malgré son patronyme, n'a pas de lien de parenté avec la secrétaire de Bonaparte.

46 Sur les traductions de Freud en français voir notamment: Le Rider 2002 et Sedat 2011. 


\section{Conclusion}

Avant de devenir la proche collaboratrice d'une éminente pionnière de la psychanalyse, Anne Berman est déjà une pharmacienne propriétaire de son officine, déléguée à la chambre syndicale et membre active du mouvement Soroptimist. Elle n'a donc probablement pas besoin financièrement de trouver un poste ni même de développer son réseau social qui semble déjà tout à fait riche et diversifié. Elle va pourtant faire le choix d'embrasser une carrière de secrétaire aux perspectives incertaines. Cela lui permettra notamment d'occuper un rôle capital dans les traductions des œuvres de Freud, et ce, même si elle n'est ni psychanalyste ni traductrice professionnelle. Il s'agit alors d'un positionnement stratégique au sein de la Société Psychanalytique de Paris car nombreux sont ceux qui cherchent à prendre le contrôle de ces traductions. Mais, pour Berman, le fait de devenir la traductrice «officielle» de Freud s'inscrit probablement dans un projet professionnel plus large que celui d'exister au sein du milieu psychanalytique parisien. En tant que Soroptimist, elle aspire surtout à devenir une de ces femmes qui excellent dans son exercice professionnel.

Ses travaux de traductrice sont à la fois à l'origine de son ascension et de sa disqualification au sein du milieu psychanalytique français. On lui a ainsi reproché son manque de compétence en tant que traductrice et son absence de réel statut professionnel. Son positionnement «intermédiaire»-pas réellement expert, pas réellement amateur - a probablement fragilisé le statut de ses traductions. A la mort de la princesse de Grèce, Berman n'a plus de soutien suffisamment puissant pour participer au débat intellectuel d'égal à égal avec les membres de la Société Psychanalytique de Paris. Ses traductions vont alors être progressivement remplacées par celles produites par des personnes considérées comme plus légitimes.

Dans le cas d'Anne Berman, la condition de secrétaire particulière n'a donc pas permis d'accéder à une réelle reconnaissance scientifique par les psychanalystes, mais seulement à une légitimité partielle et fragile. Berman n'est pas pour autant une simple exécutante des volontés de la princesse de Grèce. En effet, sans être une «éminence grise», il est indéniable qu'elle a joué un rôle actif dans le mouvement psychanalytique français, notamment en défendant sa conception des traductions de l'œuvre de Freud. Ainsi, le statut de secrétaire particulier semble bien ici être une manière de faire carrière en dehors des milieux académiques ${ }^{47}$ mais il s'agit d'un positionnement hybride produisant donc un savoir dont la légitimité est aisément remise en question.

47 Carroy/Edelman/Ohayon/Richard 2005; Oertzen/Rentetzi/Watkins 2013. 


\section{Bibliographie}

Amouroux, Rémy, «AA serious venture»: John Rodker (1894-1955) and the Imago Publishing Company (1939-60)», International Journal of Psychoanalysis 92 (2011) 1437-1454

Berman, Anne, «Recherches sur la structure anatomique du fruit des borraginacées», Thèse de pharmacie (Paris 1926)

Bertin, Célia, Marie Bonaparte (Paris 1982)

Blanc-Sanchez, Mireille, «La parole confisquée: le secrétaire dans l'Italie des XVI ${ }^{\mathrm{e}}$ et XVII" siècles», Revue du Littoral 34-35 (1992) 9-28

Bourgeron, Jean-Pierre, «Anne Berman», dans: Alain de Mijolla (éd.), Dictionnaire international d'histoire de la psychanalyse (Paris 2005) 195-196.

Bouiguignon, Odile, Traduire Freud (Paris 1989)

Carroy, Jacqueline/Nicole Edelman/Annick Ohayon/ Nathalie Richard (éds.), Les femmes dans les sciences de l'homme (XIX ${ }^{\mathrm{e}}-\mathrm{XX}^{\mathrm{e}}$ siècles) (Paris 2005)

Ellenberger, Henri F., Histoire de la découverte de l'inconscient (Paris 1994)

Freud, Sigmund, Inhibition, symptôme angoisse (Paris 1951) [or. 1926]

Gardey, Delphine, «Mécaniser l'écriture et photographier la parole. Des utopies au monde du bureau, histoires de genre et de techniques», Annales. Histoire, Sciences Sociales 54 (1999) 587-614

Godin, Jean-Guy, Jacques Lacan, 5 rue de Lille (Paris 1990)

Guirimand, Nicolas, «De la réparation des «gueules cassées〉 à la 〈sculpture du visage>. La naissance de la chirurgie esthétique en France pendant l'entre-deuxguerres», Actes de la recherche en sciences sociales 156-157 (2005) 72-87

Haddad, Gérard, Le jour où Lacan m'a adopté: Mon analyse avec Lacan (Paris 2002)

Holton, Gerald, «The woman in Einstein's Shadow», in: Gavrolu Kostas/Jürgen Renn (éds), Positioning the History of Science (2007) 95-98.

Jacquemin, Jeannine, Le Soroptimist international et Suzanne Noël (Paris 1988)

Le Rider, Jacques, «Les traducteurs de Freud à l'épreuve de l'étranger», Essaim 9 (2002) 5-14

Laplanche, Jean, «Clinique de la traduction freudienne», Ecrits du temps 7 (1984) 5-14

Laplanche, Jean/Jean-Bertrand Pontalis, Vocabulaire de la psychanalyse (Paris, 1967)

Lieberman, E. James, Acts of Will: The Life and Work of Otto Rank (New York 1985)

Marinelli, Lydia/Andreas Mayer, Rêver avec Freud. L'histoire collective de "L'interprétation du rêve» (Paris 2009)

Mijolla, Alain de, «L'édition en français des œuvres de Freud avant 1940. Autour de quelques documents nouveaux», Revue internationale d'histoire de la psychanalyse 4 (1991) 209-270

Noël, Suzanne, La Chirurgie esthétique, son rôle social (Paris 1926)

Oertzen, Christine von, «Science in the cradle: Milicent Shinn and her home-based network of baby observers», Centaurus 55 (2013) 175-195

Oertzen, Christine von/Maria Rentetzi/Elisabeth S. Watkins, «Finding science in surprising places: Gender and the geography of scientific knowledge introduction to Beyond the academy: Histories of gender and knowledge», Centaurus 55 (2013) $73-80$ 
Ohayon, Annick, L'impossible rencontre. Psychologie et psychanalyse en France (1919-1969) (Paris 1999)

Pierre, Benoît, Le Père Joseph, l'éminence grise de Richelieu (Paris 2007)

Pierrakos, Maria, La «tapeuse» de Lacan. Souvenirs d'une sténotypiste fâchée. Réflexions d'une psychanalyste navrée (Paris 2003)

Pigeard-Micault, Natalie, Les femmes du laboratoire de Marie Curie (Paris 2013)

Pinto, Josiane, «Une relation enchantée: La secrétaire et son patron», Actes de la recherche en sciences sociales 84 (1990) 32-48

Poisson, Guillaume, «Le rôle des secrétaires-interprètes de l'ambassadeur de France à Soleure dans la seconde moitié du XVII ${ }^{e}$ siècle», Etude de lettres 3 (2010) $137-154$

Pomata, Gianna, «Amateurs by Choice: Women and the Pursuit of Independent Scholarship in $20^{\text {th }}$ Century Historical Writing», 55 Centaurus (2013) 196-219

Rey Pierre, Une saison chez Lacan (Paris 1989)

Roudinesco, Elisabeth, Histoire de la psychanalyse en France. 2 (Paris 1994)

Sédat, Jacques, «La réception de Freud en France durant la première moitié du XX ${ }^{\mathrm{e}}$ siècle. Le freudisme à l'épreuve de l'esprit latin», Topique 115 (2011) 51-68

Soroptimist-Club, «Dîner du 22 mai», Bulletin de l'Union nationale des SoroptimistClubs de France 3 (1928) 3-4

Soroptimist-Club, «Liste des membres», Bulletin de l'Union nationale des Soroptimist-Clubs de France 12 (1947) 12-17 